\title{
Structural investigation of neutron-deficient Pt isotopes: the case of ${ }^{178} \mathbf{P t}$
}

Christoph Fransen ${ }^{1, *}$, Franziska Mammes ${ }^{1}$, Robert Bark ${ }^{2}$, Thomas Braunroth ${ }^{1}$, Zinhle Buthelezi ${ }^{2}$, Alfred Dewald ${ }^{1}$, Tshepo Dinoko ${ }^{2}$, Siegie Förtsch ${ }^{2}$, Matthias Hackstein ${ }^{1}$, Jan Jolie ${ }^{1}$, Pete Jones ${ }^{2}$, Elena Lawrie ${ }^{2}$, Julia Litzinger ${ }^{1}$, Claus Müller-Gatermann ${ }^{1}$, Richard Newman ${ }^{3}$, Nima Saed-Samii ${ }^{1}$, John F. Sharpey-Schafer ${ }^{2,4}$, Obed Shirinda ${ }^{2}$, Ricky Smit ${ }^{2}$, Nigel Warr ${ }^{1}$, Mathis Wiedeking ${ }^{2}$, and Karl-Oskar Zell ${ }^{1}$

${ }^{1}$ Institut für Kernphysik, Universität zu Köln, Zülpicher Strasse 77, 50937 Cologne, Germany

${ }^{2}$ iThemba LABS, National Research Foundation, P.O.Box 722, Somerset West, 7129, South Africa

${ }^{3}$ Department of Physics, Stellenbosch University, Matieland, Stellenbosch 7602, South Africa

${ }^{4}$ Department of Physics, University of the Western Cape, Robert Sobukwe Road, Bellville 7535, South Africa

\begin{abstract}
Lifetime measurements with the recoil distance Doppler-shift technique have been performed to determine yrast $E 2$ transition strengths in ${ }^{178} \mathrm{Pt}$. The experimental data are related to those on neighboring $\mathrm{Pt}$ isotopes, especially recent data on ${ }^{180} \mathrm{Pt}$, and compared to calculations within the interacting boson model and a Hartree-Fock Bogoliubov approach. These models predict prolate deformed ground states in Pt isotopes close to neutron midshell consistent with the experimental findings. Further, evidence was found that the prolate intruder structure observed in neutron-deficient $\mathrm{Hg}$ isotopes that is minimum in energy in ${ }^{182} \mathrm{Hg}$ becomes the ground state configuration in ${ }^{178} \mathrm{Pt}$ and neighboring ${ }^{180} \mathrm{Pt}$ with nearly identical transition quadrupole moments. The new data on ${ }^{178} \mathrm{Pt}$ are further discussed in the context of the systematics along the Pt isotopic chain with respect to a possible sharp shape transition towards a weakly deformed or a quasi-vibrational ground state whereas the prolate structure increases in energy in ${ }^{174,176} \mathrm{Pt}$ and becomes an intruder configuration similar to, for example, ${ }^{180,182} \mathrm{Hg}$.
\end{abstract}

\section{Introduction}

Neutron deficient nuclei in the $A=180$ mass region close to the $Z=82$ main shell closure are the subject of numerous experimental and theoretical studies. Here, challenges are the understanding of both the shape evolution for isotopic chains where the proton number is not far from magicity and the phenomenon of shape coexistence that is very predominant in $\mathrm{Pb}$ and $\mathrm{Hg}$ isotopes close to neutron midhell (see, e.g., [1] for a general overview and $[2,3]$ for experimental data on $\mathrm{Pb}$ and $\mathrm{Hg}$ isotopes, respectively). In $\mathrm{Hg}$ isotopes close to neutron midshell, i.e., around $N=104$, clear evidence was found for a weakly oblate ground state configuration with quite constant energy systematics and a prolate intruder band with a parabolic trend of the level energies with respect to the neutron number where minimum energies of the latter are reached for ${ }^{182} \mathrm{Hg}$ [4]. In a very recent work on ${ }^{178} \mathrm{Hg}$ [5] the expected shift of the prolate deformed structure toward higher excitation energies for decreasing neutron number was proven from $E 2$ transition strengths. In addition, it turned out that an intermediate deformation between normal and superdeformation has been attributed to the prolate intruders [5]. The latter is a new phenomenon in the chain of the $\mathrm{Hg}$ isotopes.

Due to these experimental findings that are also supported by different theoretical approaches, an investigation

*e-mail: fransen@ikp.uni-koeln.de of the neighboring neutron deficient $\mathrm{Pt}$ isotopic chain is of enhanced interest. A detailed analysis of ${ }^{180} \mathrm{Pt}$ including the measurement of $E 2$ transition strengths between the lowest yrast levels indicates an axially deformed shape with slight $\gamma$ softness [6]. It is important to stress that the deformation found for the ground state configuration in ${ }^{180} \mathrm{Pt}$ is comparable to the deformation of the intruder states in ${ }^{182} \mathrm{Hg}$ as discussed in [6]. In that framework it should be noted that neighboring ${ }^{176,178}$ Os exhibit signatures for nuclei at the critical point of the transition from spherical to axialsymmetric rotor nuclei $(X(5))$ [7]. Nevertheless, ${ }^{180} \mathrm{Pt}$ does not follow the expected trend for transition quadrupole moments in the close vicinity of $\mathrm{X}(5)$ nuclei and approaching the $N=82$ shell closure. In fact, the rather large quadrupole deformations of both ${ }^{180} \mathrm{Pt}$ [6] and ${ }^{182} \mathrm{Pt}[8]$ indicate that this deviation from the expected trend is a general effect, which might be correlated to a weakening of the $Z=82$ shell closure.

For a decreasing neutron number in the lighter Pt isotopes, an evolution from a rotor-like ground state configuration towards a shape coexistence of a quasi-vibrational structure and a well-deformed rotor intruder configuration at very low spins is expected. This was already discussed by Dracoulis and coworkers in an older publication [9] and is partly supported by the level schemes. The authors of [9] point out that the yrast bands of ${ }^{176,178} \mathrm{Pt}$ show a coexistence of a well deformed 'intruder' configuration in ${ }^{176} \mathrm{Pt}$, that is falling in energy to be the main component 


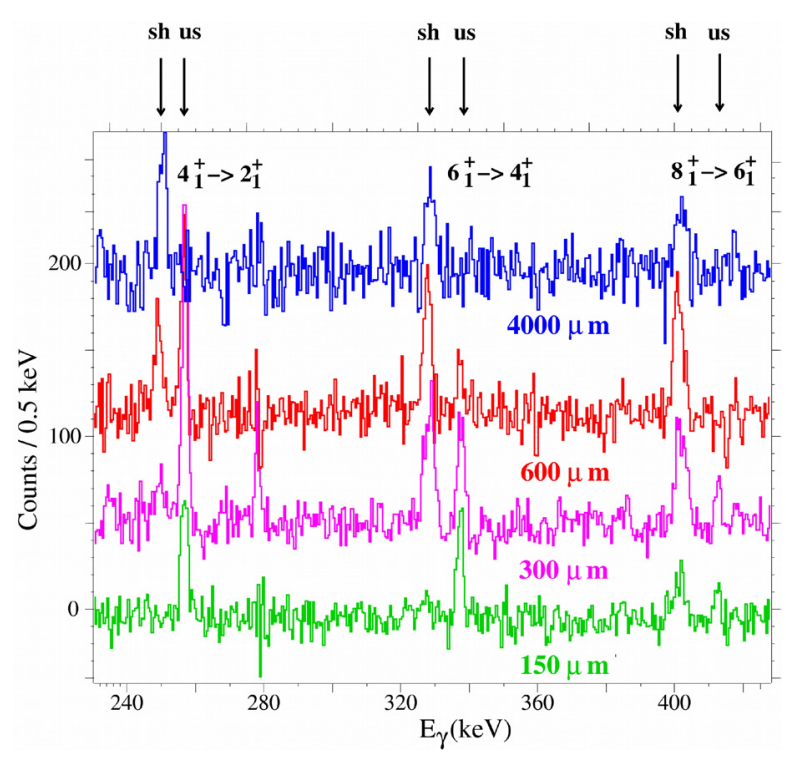

Figure 1. $\gamma$-ray spectra of ${ }^{178} \mathrm{Pt}$ for four different target-degrader distances energy-gated on the $2_{1}^{+} \rightarrow 0_{1}^{+}$transition. The labels "us" and "sh" denote the unshifted and shifted components of the respective $\gamma$-ray lines.

in the ground state of the heavier Pt isotopes as is the case for ${ }^{180} \mathrm{Pt}$ and as was observed by [6]. In addition, the very similar and nearly constant transition quadrupole moments within the yrast band of ${ }^{180} \mathrm{Pt}$ and between the higher yrast states in ${ }^{180,182} \mathrm{Hg}$ that belong to the prolate intruder configuration of $Q_{t} \approx 8 e b$ further support this picture. The prolate ground state configuration of ${ }^{180} \mathrm{Pt}$ is also evident from theoretical approaches, e.g., with the Interacting Boson Model (IBM) Hamiltonian derived from the Gogny energy density functional [10] and with the IBM with configuration mixing (IBM-CM) and a self-consistent meanfield Hartree-Fock-Bogoliubov (HFB) approach, starting from the Gogny-D1S interaction [11]. Even though these calculations explain the experimental observations very well, there are deviating results for the lighter even-even Pt isotopes from ${ }^{178} \mathrm{Pt}$ down to ${ }^{172} \mathrm{Pt}$ as pointed out in [11]: The IBM-CM calculation predicts a smooth transition to a weakly prolate deformation for decreasing neutron number towards ${ }^{172} \mathrm{Pt}$. In contrast, the self-consistent meanfield HFB approach hints at a transition from a prolate structure in ${ }^{178} \mathrm{Pt}$ via a coexistence of prolate deformation and a $\gamma$-soft structure towards spherical shape in ${ }^{172,174} \mathrm{Pt}$. Before this work experimental data were too sparse to clarify this situation.

As ${ }^{178} \mathrm{Pt}$ is expected to be just at the edge of the aforementioned transition towards a suggested shape coexistence an investigation of this nucleus with respect to the precise determination of yrast $E 2$ transition strengths is of high interest and was done in this work. So far, for ${ }^{178} \mathrm{Pt}$ level lifetimes and thus $E 2$ transition strengths were only known for the $4_{1}^{+}$and $6_{1}^{+}$states from an experiment with a single Compton-suppressed Ge detector where six $\mathrm{NaI}(\mathrm{Tl})$ detectors were used as a multiplicity trigger [9]. The authors of this publication also give a (tentative) life- time value for the $8_{1}^{+}$state but state that the respective $\gamma$ ray line is contaminated. Due to the fact that no gating on populating transitions of the levels of interest in ${ }^{178} \mathrm{Pt}$ was possible the results from [9] might be influenced by unobserved delayed sidefeeding. The lifetime of the $2_{1}^{+}$state is well known from a more recent fast-timing experiment [12].

\section{Experimental details}

To populate excited states in ${ }^{178} \mathrm{Pt}$, the ${ }^{98} \mathrm{Mo}\left({ }^{84} \mathrm{Kr}, 4 \mathrm{n}\right){ }^{178} \mathrm{Pt}$ reaction was used. The ${ }^{84} \mathrm{Kr}$ beam was provided by the $K=200$ separated sector cyclotron (SSC) at iThemba LABS, South Africa. The target consisted of an enriched, self-supporting $0.9 \mathrm{mg} / \mathrm{cm}^{2}{ }^{98} \mathrm{Mo}$ foil. After the fusionevaporation reaction, the reaction products were stopped in a $15 \mathrm{mg} / \mathrm{cm}^{2}$ gold foil. During the flight in vacuum the ${ }^{178} \mathrm{Pt}$ nuclei had a mean velocity of about $4.2 \%$ of the speed of light. Target and stopper foils were mounted in the Cologne coincidence plunger device [13] to employ the Recoil Distance Doppler-shift (RDDS) method for the determination of level lifetimes. This plunger device was used for the first time at iThemba LABS. The target-tostopper distance was monitored by measuring the capacitance between the foils. Beam-induced drifts were compensated with a feedback system using a piezoelectric device. The plunger device was coupled to the AFRODITE Ge-detector array [14]. In this experiment, it comprised of 8 escape suppressed high-purity germanium (HPGe) Clover detectors mounted in two rings with average angles of 45 and 135 degrees with respect to the beam axis. Furthermore, a low energy photon spectrometer (LEPS) detector was mounted at an angle of 90 degrees with repect to the beam axis.

Data were taken at 14 target-to-stopper distances ranging from nearly electrical contact of the foils up to 7.2 $\mathrm{mm}$. Each distance was measured for about 10 hours with an average ${ }^{84} \mathrm{Kr}$ beam current of $2.0-2.5 \mathrm{pnA}$. The reaction cross section for the $4 \mathrm{n}$ channel to ${ }^{178} \mathrm{Pt}$ was only of the order of $1 \mathrm{mb}$. Nevertheless, it was possible to analyze the data by gating on the Doppler-shifted component of each direct feeding transition of the respective level of

Table 1. Results of the RDDS experiment on the lowest yrast states in ${ }^{178} \mathrm{Pt}$ giving the level energies $E_{\text {lev }}$ (from [16]), the level lifetimes and the $E 2$ decay transition strengths $B\left(E 2 ; I_{1}^{+} \rightarrow J_{1}^{+}\right)$ $(J=I-2)$ and transition quadrupole moments derived from these observables. The more precise lifetime value of the $2_{1}^{+}$ state labeled with "*" was taken from [12] and used for the determination of the respective $E 2$ transition strength.

\begin{tabular}{ccccc}
\hline$I_{1}^{+}$ & $\begin{array}{c}E_{\text {lev }} \\
(\mathrm{keV})\end{array}$ & $\begin{array}{c}\tau\left(I_{1}^{+}\right) \\
(\mathrm{ps})\end{array}$ & $\begin{array}{c}B\left(E 2 ; I_{1}^{+} \rightarrow J_{1}^{+}\right) \\
(\text {W.u. })\end{array}$ & $\begin{array}{c}Q_{t} \\
(\mathrm{eb})\end{array}$ \\
\hline $2_{1}^{+}$ & 170.3 & $445(100)$ & & \\
& & $412(30)^{*}$ & $143_{-10}^{+11}$ & $6.53_{-0.22}^{+0.26}$ \\
$4_{1}^{+}$ & 427.4 & $40.8(24)$ & $259_{-14}^{+16}$ & $7.36_{-0.21}^{+0.23}$ \\
$6_{1}^{+}$ & 765.2 & $11.9(11)$ & $245_{-21}^{+25}$ & $6.81_{-0.29}^{+0.35}$ \\
$8_{1}^{+}$ & 1178.3 & $3.79(50)$ & $289_{-34}^{+44}$ & $7.24_{-0.42}^{+0.55}$ \\
$10_{1}^{+}$ & 1661.3 & $1.84(82)$ & $276_{-85}^{+222}$ & $7.0_{-1.1}^{+2.8}$ \\
\hline
\end{tabular}




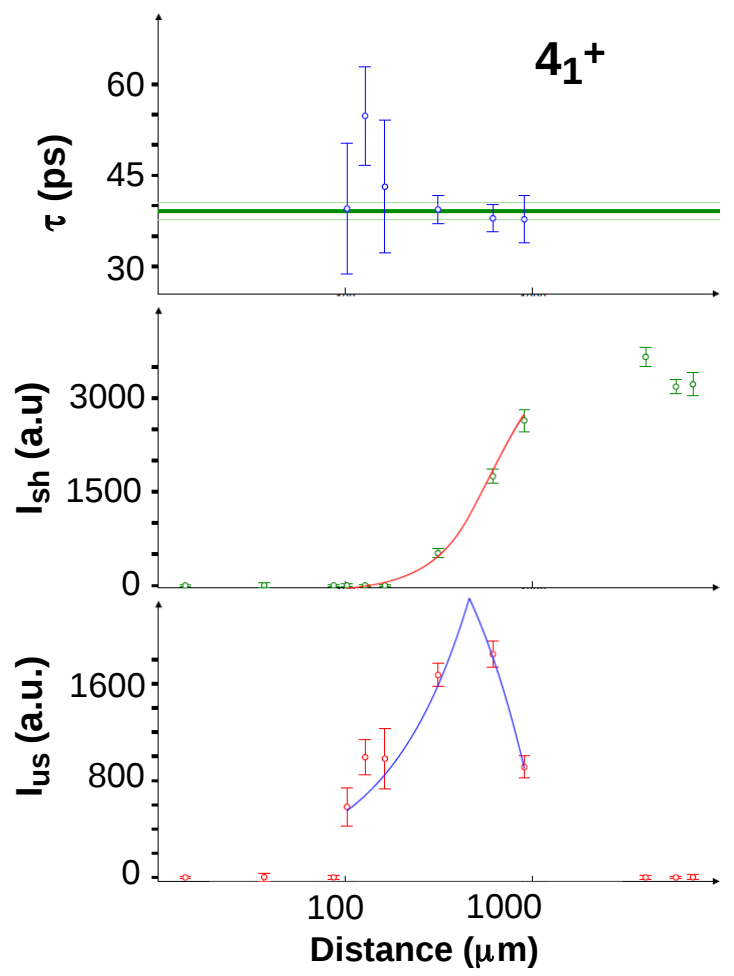

Figure 2. $\tau$-curve of the $4_{1}^{+}$state in ${ }^{178} \mathrm{Pt}$ determined from a gate on the flight component of the feeding $6_{1}^{+} \rightarrow 4_{1}^{+}$transition (upper panel). The lower two panels show the intensities of the shifted $\left(I_{s h}\right.$, middle panel) and unshifted $\left(I_{u s}\right.$, lower panel) components.

interest to exclude contributions from unoberved delayed sidefeeding.

The listmode data with $\gamma \gamma$ coincident events between all Clover HPGe detectors data were sorted into four $\gamma \gamma$ matrices for all detector-angle combinations for each distance. Each Clover was used as a single HPGe detector, i.e., the spectra of the respective four HPGe segments were summed up including an addback of Compton scattered $\gamma$ rays between the segments of the respective Clover. An example for the $\gamma$-ray spectra of this measurement showing the unshifted and Doppler-shifted components of the lowest yrast transitions in coincidence to the $2_{1}^{+} \rightarrow 0_{1}^{+} \gamma$ ray transition for four target to stopper distances is shown in Fig. 1.

The lifetimes were determined with the well established Differential Decay Curve Method (DDCM) [13, 15]. For $\gamma \gamma$-coincidence data the relation between the lifetime $\tau_{(D)}$ for a distance $D$ between target and stopper is given by

$$
\tau_{(D)}=\frac{\left\{Y_{s h}, X_{u s}\right\}_{(D)}}{\frac{d}{d t}\left\{Y_{s h}, X_{s h}\right\}_{(D)}},
$$

where $\left\{Y_{s h}, X_{u s}\right\}$ and $\left\{Y_{s h}, X_{s h}\right\}$ are the intensities of the Doppler shifted (sh) and unshifted (us) components of a decay transition $X$, determined in a direct gate on the Doppler shifted component of a direct feeding transition $Y$. The decay curve $\left\{Y_{s h}, X_{s h}\right\}_{(D)}$ was obtained by fitting it with piecewise continuously differentiable second order polynomials. As an example, Fig. 2 depicts the intensities of the shifted $\left(I_{s h}\right.$, middle panel) and unshifted $\left(I_{u s}\right.$, lower panel) components of the $4_{1}^{+} \rightarrow 2_{1}^{+}$decay transition in the direct gate on the shifted component of the feeding $6_{1}^{+} \rightarrow 4_{1}^{+}$transition including polynomial fits for all relevant distances, i.e., in the sensitive range for the $4_{1}^{+}$lifetime. The upper panel of Fig. 2 shows the lifetime values deduced via equation (1).

From the data of this experiment we were able to determine lifetimes of the $2_{1}^{+}, 4_{1}^{+}, 6_{1}^{+}, 8_{1}^{+}$, and $10_{1}^{+}$yrast states in ${ }^{178} \mathrm{Pt}$ which are listed in Tab. 1 together with the yrast $E 2$ transition strengths and transition quadrupole moments $Q_{t}$ deduced from the level lifetimes. The lifetime of the $2_{1}^{+}$ state was only determined in this work with a large uncertainty due to the low intensity of the $2_{1}^{+} \rightarrow 0_{1}^{+} \gamma$-ray transition because of its rather large conversion coefficient and a contamination of the Doppler-shifted $\gamma$-ray line observed under backward angle with a $\gamma$-ray line from ${ }^{181} \mathrm{Ta}$. Therefore, the more precise value for the $2_{1}^{+}$lifetime from a fast-timing experiment of C.B. Li and coworkers [12] is given, too, and used for the determination of the $E 2$ transition strength and $Q_{t}$ value. The fact that in the work of Dracoulis and coworkers [9] significantly longer lifetimes are given for the $4_{1}^{+}$and $6_{1}^{+}$states of $\tau\left(4_{1}^{+}\right)=54.1(46) \mathrm{ps}$ and $\tau\left(6_{1}^{+}\right)=15.7(12)$ ps supports that these results are considerably influenced by unobserved delayed sidefeeding.

\section{Discussion}

Fig. 3 displays the kinetic moments of inertia for the lower yrast states up to the $10_{1}^{+}$state in ${ }^{174,176,178,180} \mathrm{Pt}$ and ${ }^{180} \mathrm{Hg}$ as a function of the frequency $\omega\left(I_{1}^{+} \rightarrow(I-2)_{1}^{+}\right)$. A comparison of these data gives hints at a very similar structure of the prolate intruder configuration in ${ }^{180} \mathrm{Hg}$ and the ground state configuration of ${ }^{178,180} \mathrm{Pt}$ : the high absolute values of the latter and only a slight increase within the yrast band can be attributed to a quite stable deformation. The small but still recognizable decrease for the $2_{1}^{+}$state in ${ }^{178} \mathrm{Pt}$ might hint at the fact that this nucleus is right at the edge of a change towards a different structure of the lowest states for lighter $\mathrm{Pt}$ isotopes. In ${ }^{176} \mathrm{Pt}$ a clear decrease of

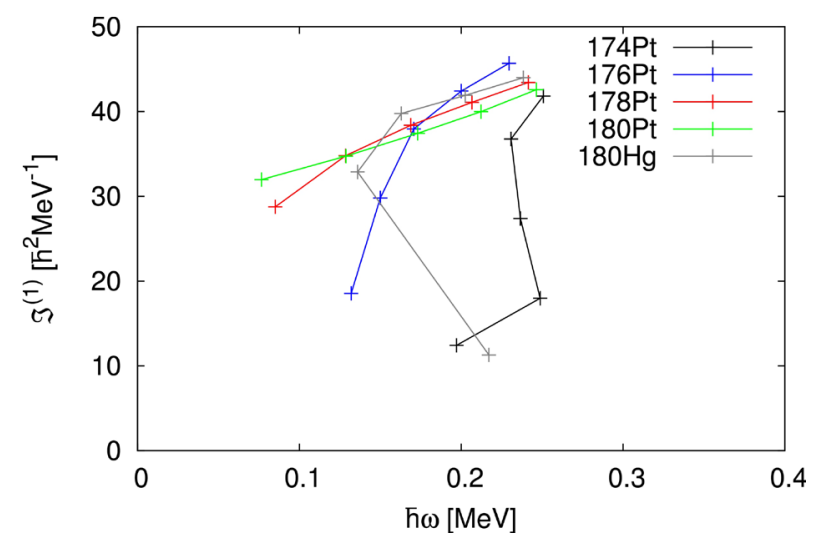

Figure 3. Kinetic moments of inertia of yrast levels of ${ }^{174-180} \mathrm{Pt}$ and ${ }^{180} \mathrm{Hg}$ up to the $10_{1}^{+}$states as a function of the frequency $\omega\left(I_{1}^{+} \rightarrow(I-2)_{1}^{+}\right)$. 
the kinetic moments of inertia is observed for both the $2_{1}^{+}$ and $4_{1}^{+}$where for ${ }^{174} \mathrm{Pt}$ this happens already for yrast states below the $10_{1}^{+}$. This is consistent with a different shape of the lower yrast states as compared to the rotational picture for ${ }^{178,180} \mathrm{Pt}$ and the higher yrast states in ${ }^{174,176} \mathrm{Pt},{ }^{180} \mathrm{Hg}$ and neighboring $\mathrm{Hg}$ isotopes as discussed in detail in [5]. This is also evidenced by a comparison of the transition quadrupole moments for ${ }^{178,180} \mathrm{Pt}$ and ${ }^{180,182} \mathrm{Hg}$ shown in Fig. 4. For ${ }^{174} \mathrm{Pt}$ no such data are available to date and for ${ }^{176} \mathrm{Pt}$ the older data from [9] could be questionable due to possible unobserved delayed sidefeeding similar to the data for ${ }^{178} \mathrm{Pt}$ from the same work.

From mixing calculations for $\mathrm{Hg}$ isotopes with $A \geq$ 180 it turned out that the wave function of the $2_{1}^{+}$state contains a significant part of the prolate intruder configuration while the ground state is weakly oblate deformed. This reduces the transition probability of the $2_{1}^{+} \rightarrow 0_{1}^{+}$transition as pointed out in [5] (see also [3, 4, 18]).

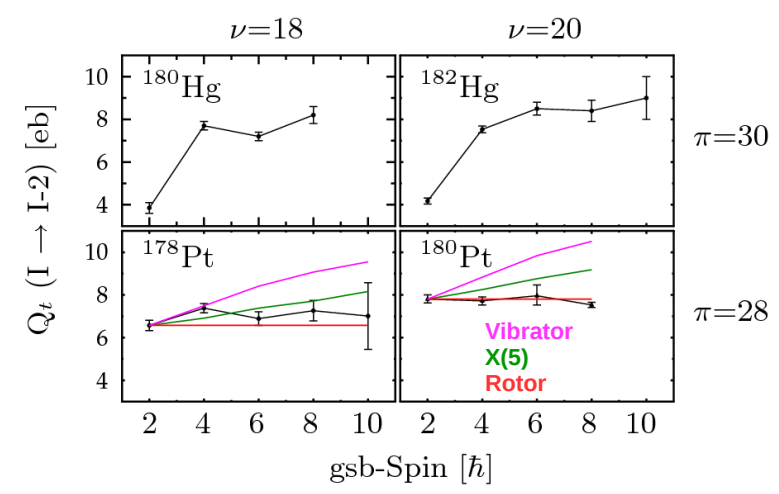

Figure 4. Transition quadrupole moments between the lowest yrast states in ${ }^{180} \mathrm{Hg}$ [4], ${ }^{182} \mathrm{Hg}$ [17], ${ }^{178} \mathrm{Pt}$ (this work and [12]) and ${ }^{180} \mathrm{Pt}[6,19]$.

For states higher than the $4_{1}^{+}$state the constant transition quadrupole moments between the corresponding yrast states of $Q_{t} \approx 8 e b$ give evidence for a rotational structure. The very similar $Q_{t}$ values within the yrast bands of both ${ }^{178,180} \mathrm{Pt}$ down to the ground state and the agreement with the expectation values for a rotor nucleus from a geometrical model prove that the prolate structure observed as an intruder structure in neutron deficient $\mathrm{Hg}$ isotopes represents the ground state configuration in ${ }^{178} \mathrm{Pt}$ in agreement with the data on the kinetic moments of inertia. However, it should be noted that the systematically slightly smaller yrast $Q_{t}$ values in ${ }^{178} \mathrm{Pt}$ hint for a slight decrease of the collectivity of the yrast states as compared to ${ }^{180} \mathrm{Pt}$.

Nevertheless, the approaches made for ${ }^{180} \mathrm{Pt}$ in [6] within the IBM with extended $Q$ formalism (see [20]) and the general collective model (GCM) [21] allowing to interprete this nucleus as a rotor nucleus with slight $\gamma$ softness apply for ${ }^{178} \mathrm{Pt}$, too, due to its very similar characteristics.
This is also in agreement with calculations with, e.g., the IBM-CM and the self-consistent mean field HFB approach [11] that were alluded in section 1 . These predict a rotor like structure of ${ }^{178} \mathrm{Pt}$ and a rapid structural change is expected for lighter $\mathrm{Pt}$ isotopes where contradicting theoretical approaches exist to date that cannot be tested in detail due to sparse experimental data. This highly motivates a measurement of yrast $E 2$ transition strengths in the lighter Pt isotopes, especially in ${ }^{174,176} \mathrm{Pt}$ which are expected to be just at the edge of this change towards a potential shape coexistence in ${ }^{176} \mathrm{Pt}$ as is supported by the kinetic moments of inertia of the lowest yrast states (see Fig. 3).

This work was supported by the Deutsche Forschungsgemeinschaft (DFG) under Contracts No. FR 3276/1-1 and DE 1516/3-1 and in part by the National Research Foundation of South Africa.

\section{References}

[1] K. Heyde, J.L. Wood, Rev. Mod. Phys. 83, 1467 (2011)

[2] A.N. Andreyev et al., Nature 405, 430 (2000)

[3] L.P. Gaffney et al., Phys. Rev. C 89, 024307 (2014); Erratum Phys. Rev. C 89, 059905 (2014)

[4] T. Grahn et al., Phys. Rev. C 80, 014324 (2009)

[5] C. Müller-Gatermann et al., Phys. Rev. C 99, 054325 (2019)

[6] C. Müller-Gatermann et al., Phys. Rev. C 97, 024336 (2018)

[7] A. Dewald et al., J. Phys. (London) G 31, S1427 (2005)

[8] K.A. Gladnishki et al., Nucl. Phys. A 877, 19 (2012)

[9] G.D. Dracoulis et al., J. Phys. G: Nucl. Phys. 12, L97 (1986)

[10] K. Nomura et al., Phys. Rev. C 83, 014309 (2011)

[11] J.E. Garcia-Ramos, K. Heyde, L.M. Robledo, R. Rodriguez-Guzman, Phys. Rev. C 89, 034313 (2014)

[12] C.B. Li et al., Phys. Rev. C 90, 047302 (2014)

[13] A. Dewald, O. Möller, P. Petkov, Prog. Part. Nucl. Phys. 67, 786 (2012)

[14] R.A. Bark et al., J. Phys. G 31, S1747 (2005)

[15] A. Dewald, S. Harissopulos, P. von Brentano, Z. Phys. A Atomic Nuclei 334, 163 (1989)

[16] E. Achterberg, O. A. Capurro, G. V. Marti, Nucl. Data Sheets 110, 1473 (2009)

[17] M. Scheck et al., Phys. Rev C 81, 014310 (2010)

[18] N. Bree et al., Phys. Rev. Lett. 112, 162701 (2014)

[19] C. Müller-Gatermann et al., Nucl. Inst. Meth. Phys. Res. A 920, 95 (2019)

[20] D.D. Warner, R.F. Casten, Phys. Rev. C 28, 1798 (1983)

[21] G. Gneuss, W. Greiner, Nucl. Phys. A 171, 449 (1971) 